Fetal Diagnosis and Therapy
Fetal Diagn Ther 2012;32:17-21

DOI: $\underline{10.1159 / 000335357}$
Received: June 17, 2011

Accepted after revision: November 26, 2011

Published online: July 6, 2012

\title{
Fetal Cardiac Function: M-Mode and 4D Spatiotemporal Image Correlation
}

\author{
M.E. Godfrey ${ }^{a} \quad$ B. Messing ${ }^{b} \quad$ D.V. Valsky ${ }^{b} \quad$ S.M. Cohen ${ }^{b} \quad$ S. Yagel ${ }^{b}$ \\ ${ }^{a}$ Department of Pediatric Cardiology, Schneider Children's Medical Center Israel, Petach Tikva, and \\ ${ }^{\mathrm{b}}$ Department of Obstetrics and Gynecology, Hadassah-Hebrew University Medical Centers, Jerusalem, Israel
}

\begin{abstract}
Key Words
M-mode $\cdot 4 \mathrm{D}$ spatiotemporal image correlation $\cdot 3 \mathrm{D} / 4 \mathrm{D}$

ultrasound · Stroke volume · Cardiac output · Fetal cardiac evaluation
\end{abstract}

\begin{abstract}
Fetal cardiac function has been shown to be a marker of disease severity in many conditions, including intrauterine growth restriction, congenital heart defects or disease (CHD), twin-to-twin transfusion syndrome, idiopathic hydrops, fetal arrhythmias, and others. It is often targeted for evaluation of patient management, for providing complete and accurate diagnoses in $\mathrm{CHD}$, for patient counseling, or in referring for fetal cardiac interventions. In this review we will describe the use of M-mode and 4D spatiotemporal image correlation in fetal cardiac evaluation, the parameters that can be measured, and their application to clinical practice.
\end{abstract}

Copyright $\odot 2012$ S. Karger AG, Basel

\section{Introduction}

Fetal cardiac function has been shown to be a marker of disease severity in many conditions, including intrauterine growth restriction, congenital heart defects or disease (CHD), twin-to-twin transfusion syndrome, idiopathic hydrops, fetal arrhythmias, and others. Cardiac evaluation is applied in considerations of patient manage-

\section{KARGER}

Fax +4161306 1234

E-Mail karger@karger.ch

www.karger.com
(C) 2012 S. Karger AG, Basel

$1015-3837 / 12 / 0322-0017 \$ 38.00 / 0$

Accessible online at:

www.karger.com/fdt ment, e.g. optimizing delivery timing, in providing complete and accurate diagnoses in $\mathrm{CHD}$, in providing prognoses for patient counseling, and, more recently, in referring for fetal cardiac interventions.

Many parameters have been devised to evaluate fetal cardiac function. Most are borrowed from adult or pediatric cardiology, such as stroke volume (SV), cardiac output (CO), ejection fraction (EF), shortening fraction (SF), and myocardial performance index.

Various modalities are applied to obtain these parameters, including the following: 3D/4D ultrasound (US) to obtain ventricular volumes to calculate $\mathrm{SV}, \mathrm{CO}$, and $\mathrm{EF}$, $\mathrm{M}$-mode to evaluate measures such as SF or the annular displacement of the atrioventricular valves, and Doppler mapping to evaluate myocardial performance and precordial venous indices such as flow across the ductus venosus and many other target veins.

In this review we will describe the use of M-mode and 4D spatiotemporal image correlation (STIC) in fetal cardiac evaluation, the parameters that can be measured, and their application to clinical practice.

\section{M-Mode}

M-mode, or motion-mode, ultrasonography is applied to the evaluation of the motion of the cardiac structures including valves, free walls, or the septa. The beam is produced by a single crystal rapidly alternating between 
Fig. 1. M-mode measurement of the endsystole and end-diastole dimensions (arrows) of the left ventricle. The M-mode trace is enlarged to show detail. $\mathrm{RV}=\mathrm{Right}$ ventricle; $L V=$ left ventricle.

Fig. 2. STIC M-mode measurement of annular displacement. After acquisition the volume can be adjusted to optimize the Mmode trace. The M-mode cursor is placed at the valve annulus. Pluses mark the zenith and nadir of annular displacement; arrow indicates amplitude measurement. The M-mode trace is enlarged to show detail.
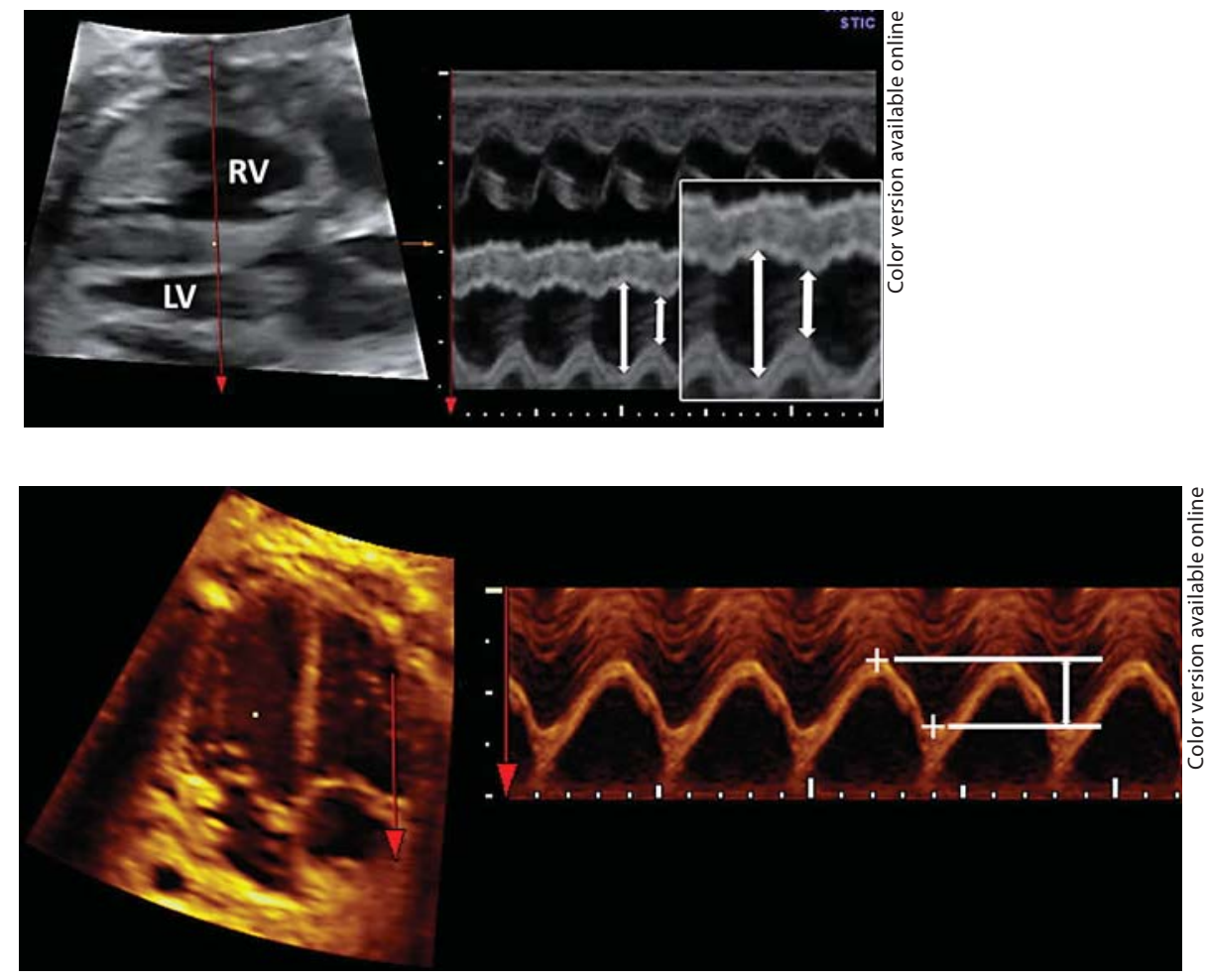

transmission and reception, updating at $>1,000 \mathrm{~Hz}$. The high temporal resolution this provides allows identification of subtle abnormalities of cardiac rhythm and determination of the relative timing of cardiac events. It is also used to evaluate cardiac dimensions such as wall thickness or chamber size. Today M-mode is usually combined with 2D US to position the beam (fig. 1).

\section{M-Mode in the Evaluation of Arrhythmia}

One of the most common applications of M-mode in the fetus is in the evaluation of fetal arrhythmia. This is achieved by aiming the beam through the atrial and ventricular walls simultaneously [1]. The M-mode cursor is placed across the anterior wall of the right ventricle, the aortic valve, and the posterior wall of the left atrium. If the fetus does not present in a position to allow this, the cursor can be placed through the right atrial wall and the posterior wall of the left ventricle [2]. The most common arrhythmias of fetal life are supraventricular tachycardia and atrial flutter. In supraventricular tachycardia, the heart rate is markedly increased, but the atria and ventricles beat at a 1:1 rate. In atrial flutter, the atrial rate may reach $300-$ $450 \mathrm{bpm}$, while the rate of the ventricles may vary widely. Some of the atrial beats are blocked while some are not, so the relative atrial and ventricular rates may be $2: 1$ or $4: 1$, for example, and this may vary over time. In fetal heart block, the atria and ventricles beat independently of each other, and this disassociation is evident in M-mode [2].

\section{Shortening Fraction}

In other situations of suspected disordered cardiac function, M-mode can be applied to obtaining measures for SF, the end-diastolic ventricle diameter and the endsystolic ventricle diameter $[\mathrm{SF}=$ (end-diastolic ventricle diameter - end-systolic ventricle diameter)/end-diastolic ventricle diameter]. The beam is positioned along the short axis of the heart in the four-chamber view, perpendicular to the interventricular septum. Normal values in the fetus have been published [3]. SF measures the difference in the end-systolic and end-diastolic diameters as a ratio to the end-diastolic diameter, and has long been considered a surrogate marker of ventricular function [4].

\section{Long-Axis Cardiac Evaluation}

M-mode can also be applied to the cardiac long axis $[5,6]$. Annular displacement measures use M-mode to quantify long-axis function as opposed to short-axis function [6]. This approach is most suited to right ven- 
tricle examination owing to the longitudinal nature of the right ventricle muscle fibers as opposed to the mainly circumferential orientation of the left ventricle muscle fibers $[6,7]$.

\section{Tricuspid Annular Displacement}

Tricuspid annular displacement is a measure of the maximum excursion of the area of junction between the tricuspid annulus and the right ventricle free wall from end-diastole to end-systole. In adults this measure of right ventricle function has been shown to have prognostic significance independent of left ventricle function [8]. In the fetus, M-mode can be applied to measure the annular displacement of mitral and tricuspid valves [5], and has been shown to increase with increasing gestational age [6]. Annular displacement uses M-mode technology that is readily available on modern US machines. However, it has not yet been validated or compared with other methods of fetal cardiac functional assessment.

The drawback in applying M-mode in the fetus stems from the problem of getting the fetus to present the heart in a position suitable for scanning and measurement, and to maintain that position long enough to obtain them.

\section{D/4D US - Spatiotemporal Image Correlation}

STIC and its place in fetal echocardiography have been reviewed in detail [9]. Briefly, STIC is based on a sweep of the fetal heart based on the five transverse plane approach [10]. The stored sweep or acquisition is a volume dataset containing a complete reconstructed cardiac cycle, consisting of approximately 1,500 images. The operator can navigate within the saved dataset both spatially and temporally using post-processing tools. From within this saved volume the operator can obtain the targeted region of interest at any stage of the cardiac cycle.

\section{STIC M-Mode}

STIC M-mode has recently been added to the post-processing tools available on machines equipped with STIC. This tool may facilitate M-mode measurements, including SF and annular displacement, by overcoming difficulties stemming from the fetal position. M-mode STIC allows adjustment of the wave pattern through the modification of image orientation: the operator can optimize the image by rotating the volume to obtain the desired plane. This modality has recently been applied to cardiac biometry [11]. STIC M-mode has also been applied to SF in normal fetuses and in cases of hydrops fetalis [12]. To

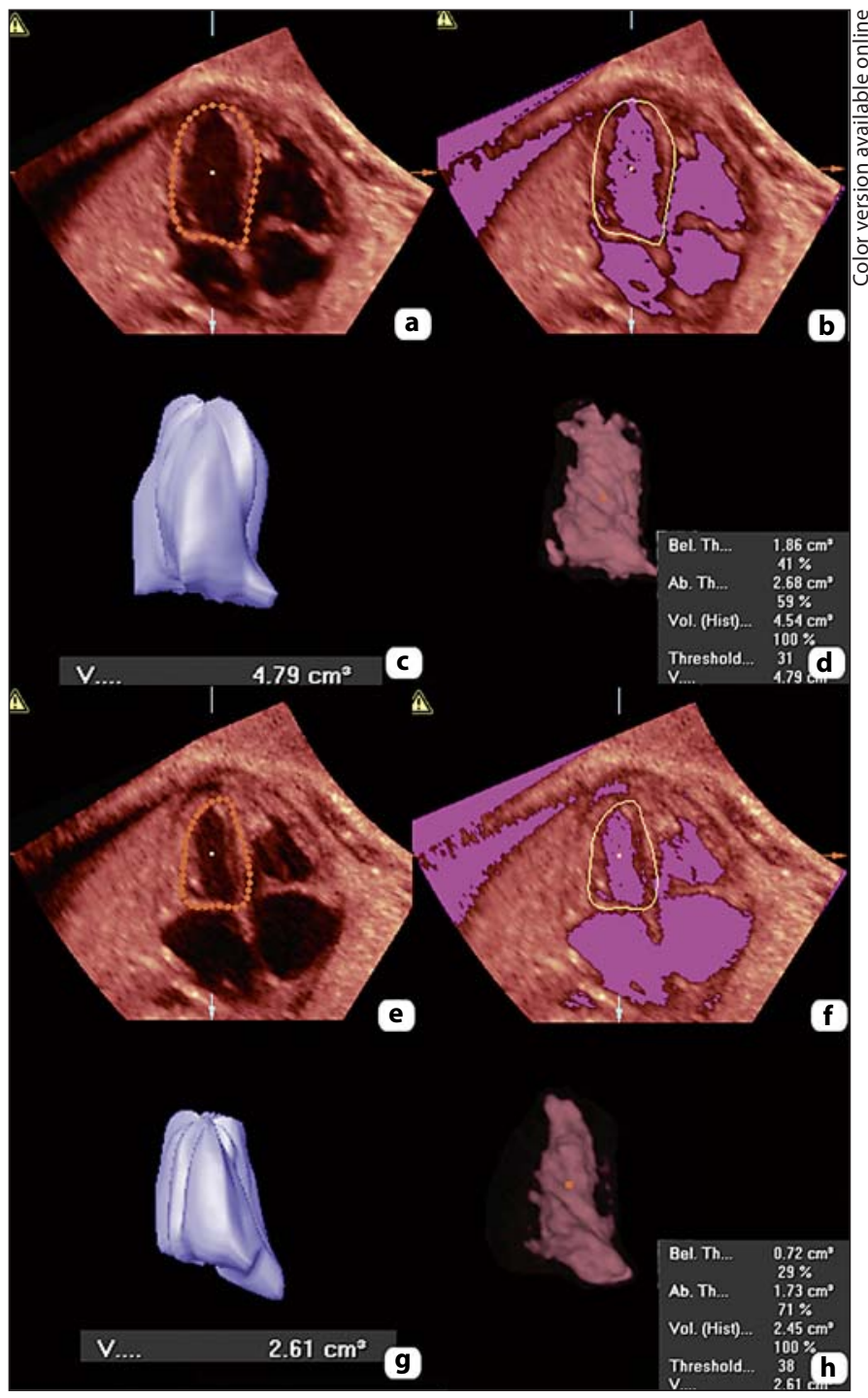

Fig. 3. Post-processing quantification of left ventricular volume in end-diastole $(\mathbf{a}-\mathbf{d})$ and end-systole $(\mathbf{e}-\mathbf{h})$. $\mathbf{a}, \mathbf{e}$ The trace in the A-frame at the level of the four-chamber view. $\mathbf{b}, \mathbf{f}$ The same frame with the IM activated. c, $\mathbf{g}$ The 3D models created by the Virtual Organ Computer-aided AnaLysis tool, which include the entire traced volume. $\mathbf{d}, \mathbf{h}$ The final intraventricular volume models. Inset Volume measurements that are then saved to the examination file [reproduced with permission from 14].

measure annular displacement, the volume is rotated and adjusted to show an apical four-chamber view, M-mode is applied to the tricuspid annulus, and the amplitude of the resulting wave is measured (fig. 2). STIC adjustment of the angle of imaging helps to guide the placement of the Mmode beam, making this a promising application, but it has not as yet been validated in large studies. 
Fig. 4. Measurement of fetal cardiac ventricle mass in a normal fetus. Automatic machine settings of threshold were used in this case. The protrusion of the moderator band into the right ventricle and the papillary muscle in the left are evident. These portions of the myocardium are included as part of the ventricle mass, with the aid of IM [reproduced with permission from 21].

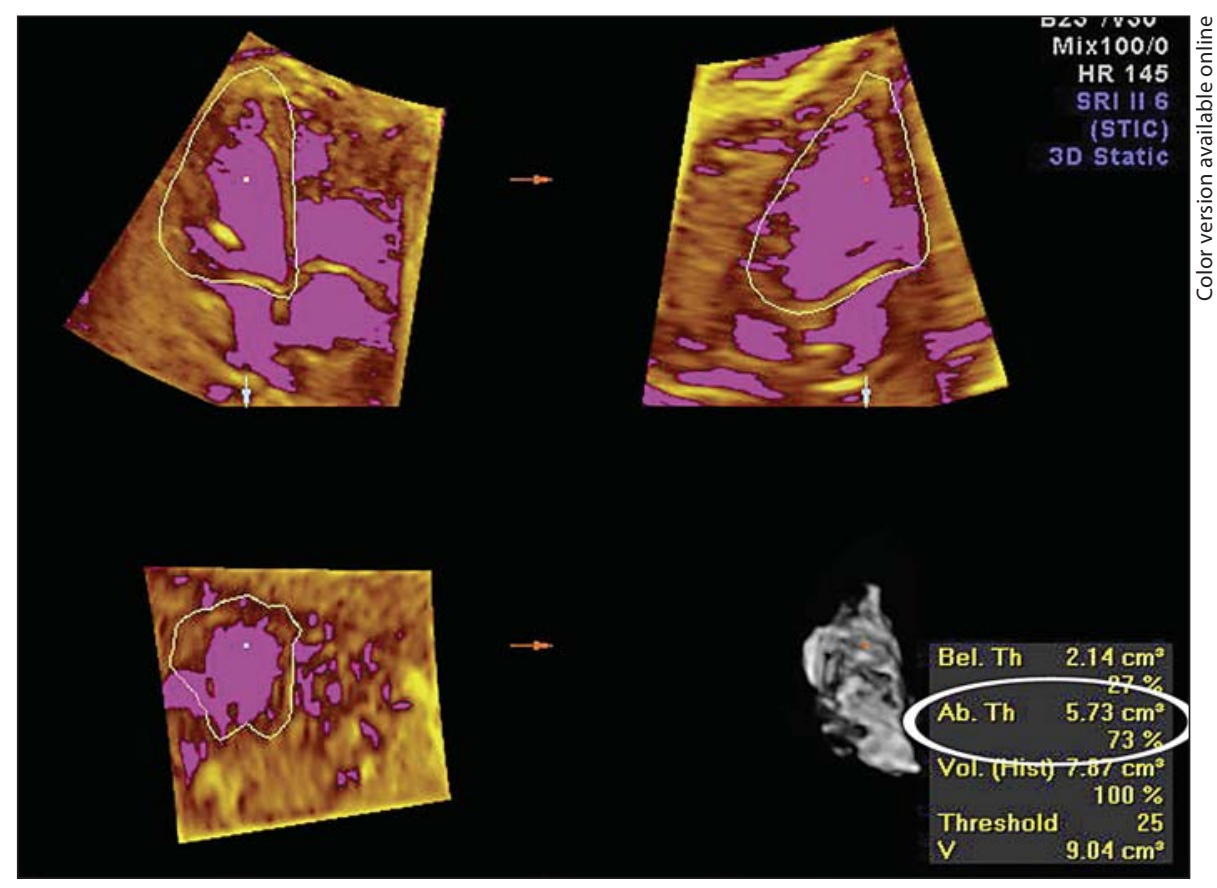

\section{Volumetry of the Fetal Heart}

By scrolling through a saved STIC volume the operator can obtain the four-chamber view at end-diastole and end-systole, identified by valve movement, and then perform either manual or semiautomated volume measurement of the cardiac ventricles. As mentioned above, many measures of cardiac function are based on cardiac ventricle volumetry (fig. 3). 3D/4D US is most often applied to fetal heart functional evaluation with the goal of obtaining ventricular volumes to extrapolate fetal SV (velocity time integral $\times$ valve area, a calculation of the volume of blood flow out of the heart at systole), EF (SV/ end-diastolic volume), and CO (SV $\times$ heart rate) [13-18]. Ventricle volumetry may be applied to the evaluation of suspected CHD with disturbance of ventricle size, such as cardiomegaly and cardiomyopathy, stenotic aortic valve and evolving hypoplastic left heart syndrome, or Ebstein anomaly.

Specialized algorithms are applied to the saved STIC volume to measure ventricle volume [13-16, 18-20]. This technique can also be extended to obtain ventricle mass [21] (fig. 4). Virtual organ computerized analysis measures the volume of a defined area by reconstructing planes around a fixed central axis. The operator can determine the number of serial traces around the axis to increase the resolution of the measurement. The applica- tion is initiated and the volume of the organ of interest is determined. Virtual organ computerized analysis has been combined with inversion mode (IM) [14, 21]. IM is a tool that isolates the fluid-filled (black) areas from tissue (gray) areas of the volume under investigation, and inverts their representation. By combining virtual organ computerized analysis and IM, the intraventricular area measured should be more accurate. The various combinations of modalities and their subcommands have been compared for feasibility and reproducibility [20].

Another approach to ventricle volumetry based on STIC volumes is manual segmentation [17]. Beginning with saved STIC volumes and scrolling through serial slices $1 \mathrm{~mm}$ apart using multiplanar reconstruction, the ventricles are measured with manual trace, and Simpson's rule is applied [17].

The disadvantages of 4D STIC are that it relies on postprocessing technologies not currently available with standard US machines. It also requires a long learning curve on the part of the operator, and there is not yet a consensus as to the optimal method of acquiring and rendering the data. However, it is a promising technique that endeavors to give the most accurate representation of the true dimensions of the fetal heart in space and time, thereby allowing calculation, rather than estimation, of SV and CO. 


\section{Conclusion}

M-mode and 4D STIC are just two of the technologies currently being used for evaluating fetal cardiac function. Indeed it could be said that they represent extremes of US technology: M-mode is a long-standing and relatively low-tech tool, while 4D STIC is a recent addition to the cutting edge of computational US technology. M-mode is convenient and quick, but can only give surrogate measurements of function. It has been widely applied clinically and reference ranges have been established for some parameters. 4D STIC is specialized and time-consuming, but in theory is capable of giving more precise results. Larger studies of its application in clinical practice are required to establish the validity of STIC-acquired measures. It will be important, as technology progresses, to carefully delineate the role each modality can play in the assessment of fetal cardiac function.

\section{References}

1 Jaeggi E: Electrophysiology for the perinatologist; in Yagel S, Silverman N, Gembruch U (eds): Fetal Cardiology. New York, Informa, 2009.

2 Simpson J, Silverman NH: Diagnosis of fetal arrhythmias during fetal life; in Yagel S, Silverman NH, Gembruch U (eds): Fetal Cardiology. London, Martin Dunitz, 2003.

$\checkmark 3$ Allan LD, Joseph MC, et al: M-mode echocardiography in the developing human fetus. Br Heart J 1982;47:573-583.

4 Quinones MA, Pickering E, et al: Percentage of shortening of the echocardiographic left ventricular dimension. Its use in determining ejection fraction and stroke volume. Chest 1978;74:59-65.

5 Carvalho JS, O'Sullivan C, et al: Right and left ventricular long-axis function in the fetus using angular $\mathrm{M}$-mode. Ultrasound $\mathrm{Ob}$ stet Gynecol 2001;18:619-622.

6 Gardiner HM, Pasquini L, et al: Myocardial tissue Doppler and long axis function in the fetal heart. Int J Cardiol 2006;113:39-47.

7 Ho SY, Nihoyannopoulos P: Anatomy, echocardiography, and normal right ventricular dimensions. Heart 2006;92(suppl 1):i2-i13.

-8 Kjaergaard J, Akkan D, et al: Right ventricular dysfunction as an independent predictor of short- and long-term mortality in patients with heart failure. Eur J Heart Fail 2007;9: 610-616.
$>9$ Yagel S, Cohen SM, et al: 3D and 4D ultrasound in fetal cardiac scanning: a new look at the fetal heart. Ultrasound Obstet Gynecol 2007;29:81-95.

10 Yagel S, Cohen SM, et al: Examination of the fetal heart by five short-axis views: a proposed screening method for comprehensive cardiac evaluation. Ultrasound Obstet Gynecol 2001;17:367-369.

11 Luewan S, Yanase Y, et al: Fetal cardiac dimensions at 14-40 weeks' gestation obtained using cardio-STIC-M. Ultrasound Obstet Gynecol 2011;37:416-422.

12 Tongsong T, Wanapirak C, et al: Fetal ventricular shortening fraction in hydrops fetalis. Obstet Gynecol 2011;117:84-91.

13 Esh-Broder E, Ushakov FB, et al: Application of free-hand three-dimensional echocardiography in the evaluation of fetal cardiac ejection fraction: a preliminary study. Ultrasound Obstet Gynecol 2004;23:546-551.

14 Messing B, Cohen SM, et al: Fetal cardiac ventricle volumetry in the second half of gestation assessed by $4 \mathrm{D}$ ultrasound using STIC combined with inversion mode. Ultrasound Obstet Gynecol 2007;30:142-151.

15 Rizzo G, Capponi A, et al: Fetal cardiac stroke volume determination by four-dimensional ultrasound with spatio-temporal image correlation compared with two-dimensional and Doppler ultrasonography. Prenat Diagn 2007;27:1147-1150.

$>16$ Molina FS, Faro C, et al: Heart stroke volume and cardiac output by four-dimensional ultrasound in normal fetuses. Ultrasound $\mathrm{Ob}$ stet Gynecol 2008;32:181-187.
17 Uittenbogaard LB, Haak MC, et al: Fetal cardiac function assessed with four-dimensional ultrasound imaging using spatiotemporal image correlation. Ultrasound Obstet Gynecol 2009;33:272-281.

18 Hamill N, Yeo L, et al: Fetal cardiac ventricular volume, cardiac output, and ejection fraction determined with 4-dimensional ultrasound using spatiotemporal image correlation and virtual organ computer-aided analysis. Am J Obstet Gynecol 2011;204:e1e10.

19 Kusanovic JP, Nien JK, et al: The use of inversion mode and 3D manual segmentation in volume measurement of fetal fluid-filled structures: comparison with Virtual Organ Computer-aided AnaLysis (VOCAL). Ultrasound Obstet Gynecol 2008;31:177-186.

20 Hamill N, Romero R, et al: Repeatability and reproducibility of fetal cardiac ventricular volume calculations using spatiotemporal image correlation and virtual organ computer-aided analysis. J Ultrasound Med 2009;28: 1301-1311.

21 Messing B, Cohen SM, et al: Fetal heart ventricle mass obtained by STIC acquisition combined with inversion mode and VOCAL. Ultrasound Obstet Gynecol 2011;38:191197. 